\title{
Discutindo a política de atenção à saúde da mulher no contexto da promoção da saúde
}

\section{Discussing the policy of attention to women's health in the context of health promoting}

\author{
Hablar de la política de atención a la salud de la mujer en el contexto de la promoción de la salud
}

Giselle Lima de Freitas', Camila Teixeira Moreira Vasconcelos", Escolástica Rejane Ferreira Moura'"I, Ana Karina Bezerra Pinheiro'v

\footnotetext{
'Enfermeira. Mestranda em Enfermagem pela Universidade Federal do Ceará (UFC). Bolsista do CNPq. Email: gisellelf@yahoo.com.br.

"Enfermeira. Especialista em Saúde Pública pela Escola de Saúde Pública do Ceará. Mestranda em Enfermagem pela UFC. Bolsista da CAPES. Email: camilamoreiravasco@hotmail.com.

II' Enfermeira. Profa. Dra. do Departamento de Enfermagem da UFC. Líder do Grupo de Pesquisa Enfermagem na Promoção da Saúde Sexual e Reprodutiva. Email: escolpaz@yahoo.com.br.

IV Enfermeira. Profa. Dra. do Departamento de Enfermagem da UFC. Líder do Projeto de Pesquisa Saúde sexual e reprodutiva: uma proposta de estudo para atividade intersetorial de promoção da saúde. Email: anakarinaufc@hotmail.com.
}

\section{RESUMO}

Na maior parte do século XX, as políticas nacionais de saúde da mulher estiveram voltadas ao ciclo gravídicopuerperal. A partir de 1984, uma proposta de atendimento integral ganhou corpo no então Programa de Assistência Integral à Saúde da Mulher (PAISM) e, em 2004, emergiu uma proposta que prioriza a promoção da saúde, por meio da Política Nacional de Assistência Integral à Saúde da Mulher (PNAISM). Com este trabalho, objetivou-se analisar, epistemologicamente, os pressupostos da promoção da saúde da PNAISM. Estudo reflexivo, baseado nos princípios e diretrizes da PNAISM e nos pressupostos da promoção da saúde apresentados nas cartas resultantes das conferências sobre promoção da saúde. A PNAISM apresenta-se de forma inovadora ao contemplar aspectos relacionados à promoção e humanização da saúde a fim de minimizar iniqüidades existentes na saúde da mulher, atendendo grupos até então alijados da sociedade e áreas antes pouco assistidas. Palavras-chave: Saúde da mulher; Promoção da saúde; Políticas Públicas de Saúde.

Descritores: Saúde da mulher; Promoção da saúde; Políticas públicas de saúde.

\section{ABSTRACT}

In most of the twentieth century, national health policies were addressed to women's pregnancy-puerperal cycle. In 1980, a proposal to care then gained in the Program for Integrated Women's Health (PAISM) and in 2004, emerged a proposal that prioritizes health promotion, through the National Policy for Integrated Health Women's (PNAISM). The purpose of this study was to analyze, epistemological, the assumptions of health promotion of PNAISM.A reflective study, based on the principles and guidelines of PNAISM and assumptions of health promotion presented in letters from conferences on health promotion. The PNAISM presents itself in an innovative way to address issues related to the promotion and humanization of health in order to minimize inequities in women's health, attending excluded groups of society and areas just before assisted.

Descriptors: Women's health; Health promotion; Health Public Policy.

\section{RESUMEN}

En la mayor parte del siglo XX, las políticas nacionales sobre la salud de la mujer se convirtieron en el embarazo y el parto. En 1984, una propuesta para la plena asistencia ganó espacio en el Programa Integrado da Salud de la Mujer (PAISM) y, en 2004, surgió una propuesta que dio prioridad a la promoción de la salud, a través de la Política Nacional de Atención Integral a la Salud de la Mujer (PNAISM). El objetivo de este estudio fue analizar, de manera epistemológica, la hipótesis de la promoción de la salud del PNAISM. Estudio de reflexión, sobre la base de los principios y directrices de la PNAISM y de la promoción de la salud presentados en las cartas de conferencias sobre la promoción de la salud. EI PNAISM se presenta en una forma innovadora de abordar las cuestiones relacionadas con la promoción y la humanización de la salud con el fin de minimizar las desigualdades en la salud de la mujer, acogiendo grupos hasta ahora excluidos de la sociedad y las zonas poco antes asistidas.

Descriptores: Salud de la mujer; Promoción de la salud; Política Públicas de Salud. 


\section{NTRODUÇÃO}

A partir das primeiras décadas do século $X X$, a atenção à saúde da mulher passou a fazer parte das políticas públicas de saúde no Brasil. No entanto, nas décadas de 30,40 e 50 a mulher era vista basicamente como mãe e "dona de casa". Na década de 60 , diversos países se voltaram para controlar a natalidade e destacou-se a atenção do Estado às mulheres em idade fértil. Assim, os programas de "controle da natalidade" ganharam destaque no final da década de 70, negando atenção às reais necessidades ou preferências das mulheres, que ficavam relegadas em segundo plano(1).

Ainda na década de 60, o movimento feminista brasileiro descontente com as diferenças de gênero e com o enfoque reducionista dado à mulher, reivindicou a não-hierarquização das especificidades de homens e mulheres, propondo igualdade social que reconhecesse as diferenças, hoje expressa na idéia de "eqüidade de gênero". Assim, emergiu um novo conceito de saúde da mulher, rompendo com o paradigma vigente centrado na função controlista da reprodução, pontuando a saúde sexual e reprodutiva como um direito ${ }^{(2)}$.

Esse engajamento das mulheres na luta pelos seus direitos e por melhores condições de vida impulsionou a adoção das primeiras medidas oficiais do Ministério da Saúde voltadas para a assistência integral à saúde da mulher. Apesar das limitações impostas pelo governo militar da época, o movimento feminista se reorganizou incitando debates que denunciavam a precariedade da saúde da mulher brasileira $^{(3)}$.

Tal precariedade referia-se, principalmente, à redução de ações que contemplavam à mulher sob um único aspecto, relacionado ao seu estado gravídico-puerperal, tendo em vista que apenas em meados da década de 80 é que foram incorporadas ações de caráter integral à saúde da mulher. Na atualidade, o conceito de saúde da mulher é amplo, contemplando os direitos humanos e a cidadania como necessidades de atenção ${ }^{(4)}$.

As mulheres em idade reprodutiva, ou seja, de 10 a 49 anos, representam $65 \%$ da população feminina brasileira. Apesar dessa predominância ainda são observadas importantes diferenças de gênero. As diferenças se traduzem nas discrepâncias salariais entre os sexos, na maior ocupação de cargos de chefias pelo sexo masculino e na violência contra a mulher, e se refletem, também, nas leis, políticas e práticas sociais que repercutem desigualdades de classe, idade, dentre outras ${ }^{(4)}$

Nesse contexto, a necessidade da integralização da atenção é percebida, sendo fortalecida com as discussões sobre a "promoção da saúde", como norte para as práticas, organização e gestão da saúde no Brasil. O termo promoção da saúde, definido em 1986, durante a I Conferência Internacional sobre
Promoção da Saúde em Ottawa, no Canadá, estava associado aos elementos da atenção primária. A responsabilidade pela saúde era exclusivamente desse setor e se baseava no modelo biomédico, voltado à atividade curativa, de forma individualizada e, portanto, de baixo impacto. Mais tarde, o termo Promoção da Saúde passou a ser associado à qualidade de vida e o sujeito passou a ter autonomia sobre seu estado de saúde ${ }^{(5)}$.

Assim, tornou-se urgente a necessidade de elaboração de políticas públicas que atendessem a mulher em todos os aspectos e não apenas no aspecto reprodutivo. Com a crescente consolidação das ações de promoção da saúde, o cenário mundial vem sendo transformado e uma ênfase é dada à visão integral dos indivíduos. Logo, vislumbrando a influência desse tema nas políticas públicas nacionais voltadas à saúde da mulher, decidiu-se pela realização deste estudo que objetiva analisar, epistemologicamente, os pressupostos da promoção da saúde na atual Política Nacional de Atenção Integral à Saúde da Mulher (PNAISM).

Trata-se de uma reflexão baseada nos princípios e diretrizes da PNAISM, lançada em 2004 e nos pressupostos da promoção da saúde apresentados nas sete cartas resultantes das conferências mundiais sobre promoção da saúde (cinco de caráter internacional: Ottawa, Adelaide, Sundsvall, Jakarta e México e uma de caráter de caráter sub-regional: Bogotá). As referidas fontes foram identificadas, lidas, fichadas e interpretadas pelas autoras no período de maio a julho de 2007, que se utilizaram um raciocínio crítico, baseado em suas próprias experiências como profissionais de saúde atuantes na área da saúde da mulher e como mulheres, bem como adotando idéias de outros autores.

\section{RESGATE HI STÓRICO}

No início do século $X X$ e até meados da década de 70, a mulher era assistida de forma restrita, reducionista e fragmentada, com ações voltadas ao ciclo gravídico-puerperal. As ações eram verticalizadas e centralizadoras, o que distanciava as medidas adotadas das reais necessidades dessa população-alvo(6). Nesse sentido, o movimento feminista iniciou uma série de reivindicações com o objetivo de incorporar às políticas de saúde da mulher outras questões como gênero, trabalho, desigualdade, sexualidade, anticoncepção e prevenção de doenças sexualmente transmissíveis ${ }^{(7)}$.

Na década de 70, foi então lançado o Programa de Saúde Materno-Infantil, no qual o planejamento familiar figurava sob o enfoque da paternidade responsável, objetivando reduzir as elevadas taxas de morbidade e de mortalidade infantil e materna( ${ }^{(8)}$. Em 1983, o governo brasileiro lançou o Programa de Assistência Integral à Saúde da Mulher (PAISM) que adotava, com dificuldade, políticas e medidas para 
permitir o acesso da população aos meios de contracepção e buscava integralizar essa assistência, incorporando medidas educativas, preventivas, de promoção, diagnóstico, tratamento e recuperação nos âmbitos da ginecologia; pré-natal, parto e puerpério; climatério; planejamento familiar; doenças sexualmente transmissíveis e câncer de mama e colo de útero ${ }^{(9-10)}$.

O Movimento da Reforma Sanitária, iniciado na década de 80, influenciou a implementação do PAISM que se caracterizou pelas propostas de descentralização, hierarquização e regionalização dos serviços $^{(8)}$.

Uma análise das ações de atenção à saúde da mulher realizada de 1998 a 2002 afirmou que apesar do discurso integralizador, ainda assim, as políticas públicas voltadas a essa área do cuidado, mantiveram a ênfase na resolução de problemas de ordem reprodutiva. Por outro lado, pôde ser observada a incorporação de um novo tema, a redução da violência sexual, demonstrando a preocupação dessas políticas em atender a mulher em seus aspectos mais gerais ${ }^{(11)}$.

Em 2004, o Ministério da Saúde elaborou o documento da Política Nacional de Atenção Integral à Saúde da Mulher - Princípios e Diretrizes (PNAISM). Este reflete o compromisso com a implementação de ações em saúde da mulher, garantindo seus direitos e reduzindo agravos por causas preveníveis e evitáveis, enfocando, principalmente, a atenção obstétrica, o planejamento familiar, a atenção ao abortamento inseguro e o combate à violência doméstica e sexual ${ }^{(4)}$.

\section{A PROMOÇÃo dA SAÚde NO CONTEXTO DA SAÚDE DA MULHER}

O modelo de Promoção da Saúde surgiu na década de 70 no Canadá, baseando-se em quatro pólos: a biologia humana; o sistema de organização dos serviços; o ambiente social, psicológico e físico; e o estilo de vida, que está em amplo processo de construção ${ }^{(12)}$.

A Conferência de Alma-Ata, realizada em 1978, estabeleceu em sua Declaração a atenção à assistência materno-infantil como prioridade, com inclusão do planejamento familiar, relacionado não só a aspectos procriativos, mas abrangendo o conjunto das necessidades de uma família, tais como: saúde, educação, moradia e lazer. A II Conferência Internacional sobre Promoção da Saúde, realizada em Adelaide em 1988, teve como tema central políticas públicas voltadas para a saúde. Foram apresentadas quatro áreas prioritárias para promoção de ações, e o apoio à saúde da mulher foi considerado como uma destas áreas, com destaque para a valorização da mulher trabalhadora e a igualdade de direitos na divisão do trabalho(13).
No entanto, somente em 1986 foi estabelecido o conceito de Promoção da Saúde, durante a Conferência de Ottawa, como sendo o processo de capacitação da comunidade para atuar na melhoria da qualidade de vida e saúde, incluindo uma maior participação no controle desse processo(14).

A Conferência de Sundsvall (1991) que teve como tema central a criação de Ambientes Saudáveis à Saúde, reconheceu a mulher como peça importante para a sua construção ao considerar o crescimento populacional uma ameaça ao desenvolvimento sustentável, tendo em vista a super-povoação de ambientes insalubres e o aumento da pobreza, o que incrementou a discussão sobre políticas de saúde da mulher e sobre o planejamento familiar ${ }^{(13)}$

Com o objetivo de adaptar os princípios, estratégias e compromissos relacionados ao sucesso da saúde da população à realidade dos países latinos, aconteceu a Declaração de Bogotá (1992). Partindo dos pressupostos já estabelecidos pelas conferências anteriores, este evento estabeleceu como compromisso para a promoção da saúde, a eliminação dos efeitos diferenciais da iniqüidade sobre a mulher, considerando-a como um elo indispensável na promoção da saúde na América Latina(13)

Nota-se que as políticas de incentivo e apoio à saúde da mulher representaram temáticas importantes em várias das Conferências de Saúde, apesar da valorização da fase reprodutiva observada nas primeiras discussões.

Essa retrospectiva traz à tona o olhar reducionista do conceito de saúde baseado no modelo biomédico, como ausência de enfermidades, e o conceito de doença como uma fatalidade. Assim, o advento da Promoção da Saúde propõe o surgimento de um novo paradigma que objetiva romper com a visão fatalista da doença, arraigada culturalmente, e aprofundar o conceito de saúde sob uma nova ótica(15).

Esse novo paradigma rompe a visão curativa e centralizadora do modelo biomédico até então vigente, o que por atuar com enfoque individualista, curativo e baseado na doença é de alto custo e baixo impacto. O novo paradigma proposto baseia-se na promoção da saúde e não na cura de doenças, tem caráter coletivo, de autonomia e co-responsabilidade dos sujeitos, e atua por meio de parcerias com educação, ação social e trabalho( ${ }^{(16)}$.

\section{POLÍTICA NACIONAL DE ATENÇÃO INTEGRAL À SAÚDE DA MULHER verSUS PROMOÇÃO DA SAÚDE}

Tendo em vista a necessidade de mudanças no cenário nacional referente às políticas voltadas à saúde da mulher, que englobe os pressupostos da promoção da saúde, foi formulada a PNAISM para 
nortear as ações de atenção à saúde da mulher de 2004 a 2007.

Os princípios e diretrizes dessa nova proposta foram discutidos em parceria com diversos segmentos da sociedade, em especial com o movimento de mulheres, o movimento negro e o de trabalhadores rurais, sociedades científicas, pesquisadores e estudiosos da área, organizações não-governamentais, gestores do SUS e agências de cooperação internacional $^{(4)}$. Esta foi uma iniciativa importante do Governo e que se baseia nos princípios da promoção, respeitando a autonomia dos sujeitos em questão e tornando-os co-autores de um processo decisório relevante para a categoria. Também demonstra a preocupação em adotar políticas consoantes às necessidades das mulheres brasileiras e, assim, reduzir os índices de morbidade e de mortalidade por causas preveníveis e evitáveis, o que não aconteceu na gênese das políticas anteriores.

Além disso, este documento consolida avanços no campo dos direitos sexuais e reprodutivos, com ênfase na melhoria da atenção obstétrica, no planejamento familiar, na atenção ao abortamento inseguro e no combate à violência doméstica e sexual. Propõem-se também a contemplar áreas como: prevenção e tratamento de mulheres vivendo com HIV/aids e as portadoras de doenças crônicas não transmissíveis e de câncer ginecológico ${ }^{(4)}$.

Esta nova política foi formulada tendo por base a avaliação das políticas anteriores e, a partir de então, buscou preencher as lacunas deixadas, como: climatério/menopausa; queixas ginecológicas; infertilidade e reprodução assistida; saúde da mulher na adolescência; doenças crônico-degenerativas; saúde ocupacional; saúde mental; doenças infectocontagiosas, bem como, a atenção às mulheres rurais, com deficiência, negras, indígenas, presidiárias e lésbicas.

Embora tenham sido observados avanços em relação à implementação dessas políticas, percebe-se ainda a exclusão de certos segmentos da sociedade, tendo em vista, que grupos como o das prostitutas, que representa uma população extremamente vulnerável a vários agravos, como: doenças sexualmente transmissíveis, violência sexual e a própria marginalização da sociedade, continuam alijados das propostas de atenção à saúde.

A PNAISM reforça a humanização da atenção em saúde, concebendo que humanização e qualidade da atenção são aspectos indissociáveis. Nesse sentido, é imprescindível considerar que humanizar é muito mais do que tratar bem, com delicadeza ou de forma amigável, devendo ser consideradas questões de acessibilidade ao serviço nos três níveis da assistência, provisão de insumos e tecnologias necessárias, formalização de sistemas de referência e contra-referência, disponibilidade de informações e orientação da clientela e a sua participação na avaliação dos serviços.

A mulher, a partir de então, é vista em sua integralidade, como sujeito autônomo e participativo no processo de decisão para a formulação de políticas públicas, tendo em vista que à medida que a mulher é incluída nesse processo, há garantia do atendimento de suas reais necessidades aumentando a qualidade da assistência.

\section{CONSI DERAÇÕES FI NAIS}

As políticas de atenção à saúde da mulher formuladas nacionalmente através de amplas e complexas discussões trouxeram contribuições imprescindíveis para o processo de transformação sobre o paradigma da saúde da mulher. Apesar dos avanços extraordinários, esse processo é dinâmico e acompanha a transformação da sociedade e, por isso, inexaurível. Cada política trouxe consigo um avanço singular no processo geral pela busca da saúde da mulher, conforme destacado anteriormente.

A atual PNAISM, objeto de reflexão neste estudo, apresenta-se de forma inovadora ao propor contemplar em seus princípios e diretrizes, aspectos relacionados à promoção e humanização da saúde a fim de minimizar as iniqüidades existentes na saúde da mulher.

Rompendo com o enfoque biologicista e medicalizador hegemônico nos serviços de saúde, a PNAISM adota o conceito de saúde integral propondo atender à mulher em todas as suas etapas de vida, englobando inclusive segmentos que estavam, até então, à margem dos serviços de saúde.

Todavia, percebe-se, ainda, certa valorização da doença em parte das ações propostas. Na prática, a integralidade da assistência não é efetivamente contemplada, tendo em vista que o sistema de saúde apresenta dificuldades em assistir a mulher nas áreas específicas de climatério, infertilidade, saúde mental e saúde ocupacional.

A PNAISM procurou em seus princípios e diretrizes estabelecer áreas prioritárias, enfatizando com isso, mulheres indígenas, lésbicas e presidiárias, mas, ainda assim, o próprio sistema de saúde enfrenta dificuldades em atender à mulher em tais especificidades.

Apesar do inegável avanço, é essencial questionar a real implementação desses princípios nos municípios e estados brasileiros. Mais ainda, para que isso aconteça é necessário um controle social efetivo por parte das mulheres, no que tange a implantação e avaliação dessas políticas. Vale ressaltar que a efetivação desta política depende igualmente dos gestores das unidades de saúde e dos profissionais que lidam diretamente com o público feminino, abrindo canais para o debate e para a crescente escolarização desse público-alvo. 
A valorização da mulher, enquanto ser humano e cidadã é algo destacável, e, devido a isto, recebeu um investimento diferenciado em relação às ações de saúde. Não desmerecendo os avanços obtidos, ainda estamos longe de desfrutar de forma plena dos conceitos e pressupostos da promoção da saúde da mulher, que estão contemplados, teoricamente, nos princípios e diretrizes da PNAISM.

\section{REFERÊNCI AS}

1. Mori ME, Coelho VLD, Estrella RCN. Sistema Único de Saúde e políticas públicas: atendimento psicológico à mulher na menopausa no Distrito Federal, Brasil. Cad. Saúde Pública. 2006; 22(9): 1825-33.

2. Giffin Karen. Pobreza, desigualdade e eqüidade em saúde: considerações a partir de uma perspectiva de gênero transversal. Cad. Saúde Pública. 2002; 18(Suppl): 103-12.

3. Formiga Filho JFN. Políticas de saúde reprodutiva no Brasil: uma análise do PAISM. In: Galvão L, Díaz J. Saúde sexual e reprodutiva no Brasil: dilema e desafios. São Paulo: Editora Hucitec/Population Council; 1999. p. 151-62.

4. Ministério da Saúde. Política nacional de atenção integral à saúde da mulher: princípios e diretrizes. Brasília: Ministério da Saúde; 2004.

5. Czeresnia D. O conceito de saúde e a diferença entre promoção e prevenção. In: Czeresnia D, Freitas CN. Promoção da saúde: conceitos, reflexões, tendências. Rio de Janeiro: FIOCRZ; 2003. p. 39-53.

6. Coelho MRS. Atenção básica à saúde da mulher: subsídios para a elaboração do manual do gestor municipal. [dissertation]. Salvador: Instituto de Saúde Coletiva/UFBA; 2003.

7. Moura ERF, Silva RM. Informação e planejamento familiar como medidas de promoção da saúde. Ciênc. saúde coletiva. 2004;9(4):1023-32.

8. Costa AM. Planejamento Familiar no Brasil. Bioética. 2000;4(2):209-17.

9. Ministério da Saúde. Assistência integral à saúde da mulher: bases da ação programática. Brasília: Ministério da Saúde; 1984.

10. Osis MJD, Faúndes A, Makuch MY, Mello MB, Sousa MH, Araújo MJO. Atenção ao planejamento familiar no Brasil hoje: reflexões sobre os resultados de uma pesquisa. Cad. Saúde Pública. 2006; 22(11): 2481-90.

11. Correia SO, Piola SF. Balanço 1998-2002: aspectos estratégicos, programáticos e financeiros. Brasília: Ministério da Saúde; 2003.

12. Andrade RBS, Almeida Filho, N. Halopatogênese: esboço de uma teoria geral de saúde-doença com base na promoção da saúde. In: Czeresnia D, Freitas MCN. Promoção da saúde: conceitos, reflexos e tendências. Rio de Janeiro: FIOCRUZ; 2003. p. 3953.
13. Ministério da Saúde. Promoção da saúde. Declaração de Alma-Ata. Carta de Otawa. Declaração de Adelaide. Declaração de Sundsvall. Declaração de Santafé de Bogotá. Declaração de Jacarta. Brasília: Ministério da Saúde; 2001.

14. Souza EM, Grundy E. Promoção da Saúde, epidemiologia social e capital social: inter-relações e perspectivas para a saúde pública. Cad. Saúde Pública. 2004;20(5): 1354-60.

15. Lefevre F, Lefevre AMC. Promoção da Saúde - a negação da negação. Rio de Janeiro: Vieira e Lent; 2004.

16. Moura ERF. Assistência ao planejamento familiar na perspectiva de usuárias e enfermeiros do Programa de Saúde da Família [thesis]. Fortaleza: Faculdade de Farmácia, Odontologia e Enfermagem/UFC; 2003. 133 p.

Artigo recebido em 13.05.08.

Aprovado para publicação em 25.05.09. 